\title{
¿Qué papel juegan la evitación del daño y la sensación de inacabado en los síntomas obsesivos de orden y comprobación?
}

\author{
María Roncero \\ Gertrudis Fornés-Romero \\ Universidad de Valencia
}

\begin{abstract}
RESUMEN
Numerosas investigaciones han intentado determinar las variables implicadas en la génesis y mantenimiento del Trastorno Obsesivo-Compulsivo (TOC). Recientemente, se han propuesto la evitación del daño y la sensación de inacabado como dos dimensiones nucleares motivadoras de la sintomatología obsesivo-compulsiva. El objetivo de este estudio fue analizar el papel de la evitación del daño y la sensación de inacabado en su relación con los síntomas obsesivo-compulsivos. Para ello, se realizó un Path analysis en una muestra de 267 personas de la población general (74,5\% mujeres; edad media 27,29 años). Los resultados mostraron que la asociación entre evitación del daño y los síntomas de orden y comprobación estaba mediada, en gran medida, por la sensación de inacabado, apoyando una mayor especificidad de esta última en la fenomenología TOC. Futuros estudios deberán corroborar este resultado en muestras clínicas.
\end{abstract}

Palabras Clave

Trastorno obsesivo-compulsivo; sensación de inacabado; evitación del daño; orden; comprobación.

\begin{abstract}
Several studies have attempted to establish the variables involved in the aetiology and maintenance of Obsessive Compulsive Disorder (OCD). Harm avoidance and incompleteness have been recently proposed as two core motivational dimensions of the obsessive-compulsive symptomatology. The aim of the present study was to analyse the role of harm avoidance and incompleteness in their relation to the obsessive-compulsive symptoms. Thus, a Path analysis was performed on a sample of 267 individuals from the general population ( $74,5 \%$ women; $M=27,29$ years old). Results showed that the association between harm avoidance and ordering and checking symptoms was largely mediated by the incompleteness, supporting a greater specificity of the latter in the OCD phenomenology. Future studies should confirm this finding in clinical samples.
\end{abstract}

KEYWORDS

Obsessive-compulsive Disorder; Incompleteness; Harm avoidance; Ordering; Checking. 
¿Qué papel juegan la evitación del daño y la sensación de inacabado en los síntomas obsesivos de orden y comprobación?

El trastorno obsesivo-compulsivo (TOC) es una condición psicopatológica caracterizada por la presencia recurrente de obsesiones y/o compulsiones (American Psychiatric Association, 2013). Las obsesiones pueden ser pensamientos, impulsos, imágenes y/o sensaciones intrusas y desagradables, mientras que las compulsiones son conductas que se realizan con el objetivo de reducir el malestar asociado a las obsesiones. Aunque la relación entre estos dos componentes del trastorno puede resultar en principio sencilla, la investigación ha demostrado que el TOC es un trastorno complejo y heterogéneo (García-Soriano, Belloch, \& Morillo, 2008; Lochner \& Stein, 2003). Durante varias décadas y hasta la actualidad, muchos han sido los esfuerzos puestos en intentar determinar las variables involucradas en la génesis y mantenimiento de la sintomatología obsesivo-compulsiva. Los modelos explicativos del TOC basados en aproximaciones cognitivo-conductuales señalan que el motivador central de la conducta compulsiva es el miedo a las consecuencias que subyace a las obsesiones (Rachman, 2002; Salkovskis, 1999). Según esta perspectiva, la técnica de elección para el tratamiento del TOC es la exposición con prevención de respuesta (EPR) y, de hecho, existe un gran cuerpo de estudios que ponen de manifiesto la eficacia de esta en el tratamiento del TOC (Abramowitz, 1996; Foa, Steketee, \& Milby, 1980; Lindsay, Crino, \& Andrews, 1997; Whittal, Robichaud, Thordarson, \& McLean, 2008). No obstante, las investigaciones también revelan que no todos los pacientes con TOC responden a la EPR (Foa et al., 2005). Asimismo, existe un porcentaje no desdeñable de pacientes con TOC cuyas conductas no están motivadas por el miedo a las consecuencias, evitación del daño o amenaza percibida (Tolin, Abramowitz, Kozak, y Foa, 2001). Por el contrario, la realidad clínica muestra que algunos pacientes ejecutan conductas dirigidas a compensar sentimientos y/o sensaciones de que algo no está como debería estar. Son varios los autores que han descrito fenómenos para tratar de explicar estas sensaciones, tales como "Feeling of Knowing" (Rapoport, 1991), "Just Right Perceptions" (Leckman, Walker, Goodman, Pauls, \& Cohen, 1994), "Sensory Phenomena" (Miguel et al., 2000), o "Not Just Right Experiences" (Coles, Frost, Heimberg, \& Rheaume, 2003). Rasmussen y Eisen (1992) describieron estas experiencias como sensaciones de inacabado (término "sense of incompleteness" en la literatura inglesa), y sugirieron que en el caso de los pacientes con TOC, las compulsiones se realizarían con la intención de eliminar o suprimir esa sensación de inacabado y conseguir un estado de perfección y certeza. Siguiendo la descripción de Rasmussen y Eisen, Summerfeldt (2004) propuso un modelo explicativo de la sintomatología TOC basado en dos dimensiones ortogonales que constituirían las dimensiones afectivo-motivacionales nucleares del TOC: la evitación del daño y la sensación de inacabado. Este modelo bidimensional, cuya estructura ha sido validada en muestras de población general y pacientes con TOC (Pietrefesa \& Coles, 2008; Summerfeldt, Kloosterman, Antony, \& Swindon, 2014), plantea que la evi- 
tación del daño muestra una similitud marcada en otros trastornos de ansiedad diferentes al TOC, mientras que la sensación de inacabado es única para los fenómenos obsesivo-compulsivos (Summerfeldt, 2004). Diversos estudios realizados con muestras clínicas y no clínicas revelan una alta relación entre la sensación de inacabado (y fenómenos similares surgidos a continuación) y evitación del daño con sintomatología TOC (Belloch et al., 2016; Coles et al., 2003, 2005; Cougle et al., 2013; Ecker \& Gönner, 2008; Ferrao et al., 2012; Fornés, Ruiz-Fernández, y Belloch, 2016; Ghisi et al., 2010; Taylor et al., 2014) demostrando así la importancia que ambas variables tienen en el TOC.

Sin embargo, a un nivel más específico, las investigaciones que estudian las relaciones entre las dos dimensiones propuestas como motivadoras de los síntomas obsesivo-compulsivos y las diferentes manifestaciones o dimensiones de síntomas TOC no arrojan resultados concluyentes. Algunos autores han relacionado la sensación de inacabado con la dimensión de lavado/limpieza (Tallis, 1996), mientras que otros encuentran que la sensación de inacabado mantiene relaciones significativas con la dimensión de simetría y orden (Coles et al., 2003, 2005; Ecker \& Gönner, 2008; Pietrefesa \& Coles, 2008). Este último hallazgo se encuentra en la línea de los resultados derivados del estudio de Radomsky y Rachman (2004). Asimismo, otros trabajos han hallado importantes asociaciones entre la sensación de inacabado y la dimensión de comprobación, tanto en población clínica (Ecker \& Gönner, 2008) como no clínica (Coles et al., 2003; Cougle, Fitch, Jaco- bson, \& Lee, 2013); Pietrefesa y Coles, 2008). Por su parte, los resultados encontrados para la evitación del daño parecen indicar que esta dimensión es más específica de las obsesiones (Ecker \& Gönner, 2008; Taylor et al., 2014) aunque también ha mostrado asociaciones (aunque débiles) con la dimensión de lavado/contaminación (Ecker \& Gönner, 2008). Este último dato, es similar al hallado por Calamari et al., (2006) quienes no encontraron la esperable asociación potente entre los síntomas de contaminación y la sobreestimación de la amenaza.

En definitiva, las investigaciones parecen indicar que la evitación del daño y la sensación de inacabado no son dimensiones excluyentes, sino que son factores motivacionales centrales que subyacen a la conducta obsesivo-compulsiva, y tienen una patente importancia en la explicación de las dimensiones de síntomas TOC, especialmente en los de orden y comprobación. Por tanto, el objetivo del presente estudio fue examinar, en una muestra de población general, el papel que juegan la evitación del daño y la sensación de inacabado en su relación con los síntomas de orden y comprobación. En concreto, se analizó si alguna de las dos dimensiones (evitación del daño y la sensación de inacabado) mediaba en la relación con los síntomas obsesivos de orden y comprobación. En base a los estudios llevados a cabo hasta el momento, se espera que ambas dimensiones revelen relaciones significativas con los síntomas de orden y comprobación, mostrando la sensación de inacabado una relación más específica. 


\section{Método}

\section{Participantes}

La muestra estuvo compuesta por 267 personas procedentes de la población general, de las cuales 199 eran mujeres (74,5\%). La edad media de los participantes fue de 27.29 años $(D T=0,72 ;$ rango $=18-60)$, la mayoría estaban solteros en el momento del estudio ( $n=197$, $73,8 \%)$, tenían un nivel socio-económico medio $(n=180,67,4 \%)$, y estudios superiores $(n=$ $217,81,3 \%)$.

\section{Instrumentos}

Inventario Obsesivo Compulsivo Revisado (OCl-R; Foa et al., 2002). Autoinforme formado por 18 ítems con escala de respuesta tipo Likert de 5 puntos, el cual mide el malestar asociado a los síntomas obsesivos-compulsivos. Se obtiene una puntuación total y las siguientes subescalas: lavado, comprobación, obsesiones, acumulación, neutralización y orden. Validación en población española realizada por Belloch, Roncero, García-Soriano, Carrió, Cabedo, y Fernández-Álvarez (2013). La fiabilidad encontrada en la presente muestra fue para la escala total de $a$ $=.88$, mientras que los valores para las subescalas se situaron entre $a=, 56$ y $a=, 81$.

Obsessive-Compulsive Core Dimensions Questionnaire (OC-CDQ; Summerfeldt et al., 2014). Cuestionario que evalúa dos dimensiones ortogonales propuestas como motivadoras de los síntomas obsesivo-compulsivos: evitación del daño y sensación de inacabado. Compuesto por 20 ítems cuya escala de respuesta oscila entre los valores 0 (nunca) y 4 (siempre). La validación de su estructura factorial en población española, fue llevada a cabo por Carrasco y Belloch (2013). Los índices de fiabilidad hallados en la muestra de este estudio fueron elevados (evitación del daño, $a=$ ,89 y sensación de inacabado, $a=, 88$ ).

\section{Procedimiento}

La recogida de la muestra se realizó mediante el método de bola de nieve. Para ello, se solicitó la participación voluntaria a estudiantes del grado de Psicología de la Universidad de Valencia. Los estudiantes completaron los instrumentos de evaluación en una primera sesión, y a continuación asistieron a un segundo encuentro en el cual se les entrenó para la administración del protocolo a 3 familiares y/o amigos. Todos los participantes de este estudio completaron una hoja con datos sociodemográficos, información relativa a la posible existencia de problemas de salud mental, y firmaron un consentimiento informado. Como compensación por la participación, una vez finalizada la investigación, se ofreció a los estudiantes la oportunidad de asistir a un seminario de investigación en el que se explicó con mayor profundidad el presente estudio.

\section{Análisis estadísticos}

Para analizar la relación entre las variables de estudio se realizaron correlaciones de Pearson, con el programa SPSS v.20 (SPSS, Chicago, IL, USA).

Se llevaron a cabo una serie de Path Analysis según el método de Máxima Verosimilitud por medio del coeficiente normalizado de Mardia que indica la normalidad de la distribución a nivel global. Se atendió a los siguientes índices de ajuste (Bentler \& Bonet, 1980): índice de ajuste 
comparativo (Comparative Fit Index; $\mathrm{CFI}$ ), índice de bondad ajuste (Goodness of Fit Index; GFI), el Root Mean-Square Error of Approximation (RMSEA), y para comparar entre modelos se empleó el criterio de información de Akaike (Akaike Information Criterion, AIC), siendo los valores menores indicadores de mejor ajuste, y cuanto más cercanos a cero indicación de una mayor parsimonia. Los modelos iniciales se plantearon siguiendo un criterio teórico, mientras que el test de Lagrange para liberación de parámetros se empleó con el objetivo de revisar el mejor de los modelos teóricos. Para este análisis se utilizó el programa EQS v6.1 (Bentler, 2006).

\section{Resultados}

Antes de efectuar el análisis Path se comprobó que existía una asociación significativa entre las variables propuestas. La evitación del daño se asoció significativamente con sensación de inacabado $(r=0,69, p<0,001)$, orden $(r=0,31, p<0,001)$, y con comprobación ( $r=$ $0,43, p<0,001)$. Asimismo, la sensación de inacabado mostró una asociación significativa con los síntomas de orden $(r=0,54, p<0,001)$ y comprobación $(r=0,45, p<0,001)$.
En primer lugar, se comprobó que se cumplía el supuesto de normalidad multivariada (coeficiente normalizado de Mardia =8,8026). A continuación, se propusieron dos modelos teóricos en los cuales la evitación del daño y la sensación de inacabado jugaban un papel mediador entre estos dos constructos respectivamente y los síntomas obsesivos de orden y comprobación (véase figura 1). En el Modelo A, el factor inacabado media la relación entre evitación del daño con los síntomas de orden y comprobación. Mientras que en el Modelo B, es la evitación del daño el mediador entre inacabado y los síntomas de orden y comprobación.

Como se observa en la tabla 1, los mejores índices se obtuvieron con el Modelo A. Siguiendo el test de Lagrange, se añadió un efecto directo entre evitación del daño y comprobación que mejoró los índices, acercándose razonablemente al criterio $\mathrm{CFI}, \mathrm{GFI}>0,90$ y RMSEA < 0,08 (Hu \& Bentler, 1999), y siendo el valor de AIC el más bajo y cercano a cero. En este modelo, se observó que la evitación del daño explica un $47 \%$ de la sensación de inacabado, y la sensación de inacabado a su vez explica un $29 \%$ y un $24 \%$ de los síntomas de orden y comprobación respectivamente.

Tabla 1.

Índices de ajuste para los modelos $A$ y $B$, y el modelo $A$ modificado.

\begin{tabular}{ccccccc}
\hline & X2 (df) & $\mathrm{p}$ & CFI & GFI & RMSEA & AIC \\
\hline Modelo A & $35.93(3)$ & 0.000 & 0.906 & 0.945 & 0.203 & 29.92 \\
Modelo B & $105.16(3)$ & 0.000 & 0.709 & 0.832 & 0.358 & 99.16 \\
Modelo A-modificado & $2.87(1)$ & 0.090 & 0.99 & 0.99 & 0.080 & 0.87 \\
\hline
\end{tabular}

CFI: Comparative Fit Index; GFI: Goodness of Fit Index; RMSEA: Root Mean-Square Error of Approximation; AIC: Akaike's Information Criterion. 
Modelo A

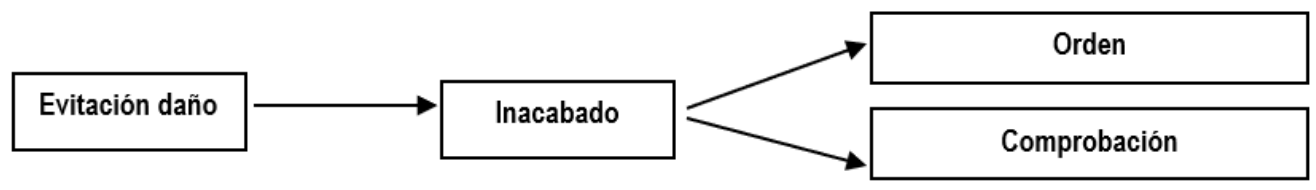

Modelo B

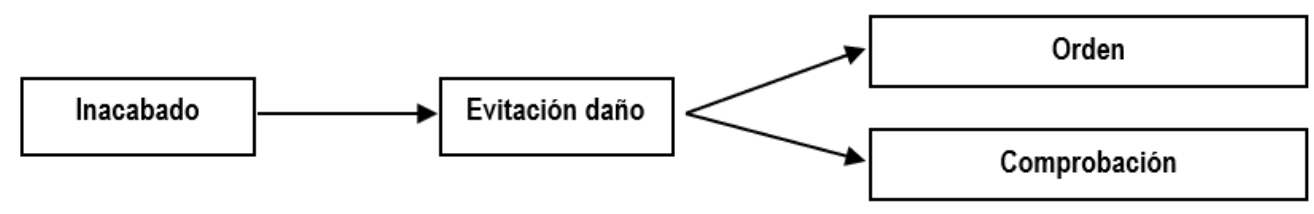

Modelo A modificado

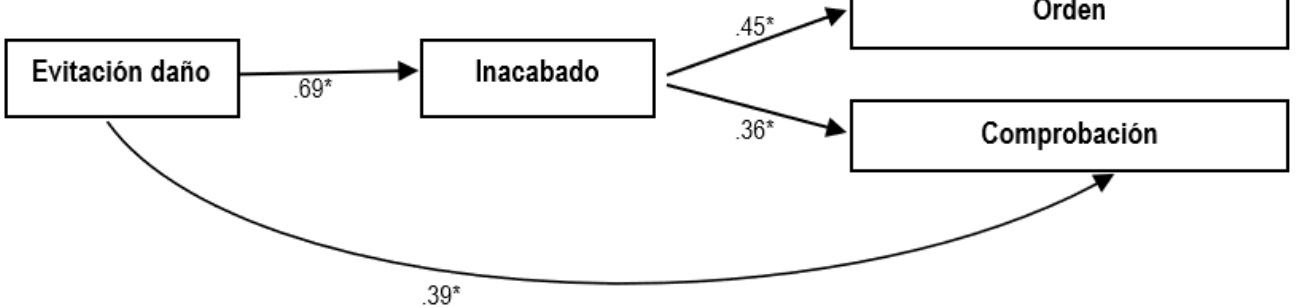

Figura 1. Modelos propuestos.

\section{Discusión}

El objetivo de este estudio fue analizar el papel de la evitación del daño y la sensación de inacabado en su relación con los síntomas de orden y comprobación en población general. Los resultados, tanto del análisis de correlación como del análisis Path, mostraron una asociación elevada entre las dos dimensiones propuestas como motivacionales de la sintomatología obsesivo-compulsiva. Este resultado es consistente con investigaciones anteriores llevadas a cabo con el mismo tipo de población (Pietrefesa \& Coles, 2008; Summerfeldt, Kloosterman, Antony, \& Swinson, 2014; Taylor et al.,
2014). Además, los resultados revelaron que ambas dimensiones se asociaron de manera significativa con los síntomas de orden y comprobación, relaciones que también se observaron en trabajos previos que aportan evidencias de la existencia de asociaciones significativas entre la sensación de inacabado y las dimensiones de orden (Coles et al., 2003; Ecker \& Gönner, 2008; Ferrão et al., 2012; Pietrefesa y Coles, 2009; Summers, Fitch, \& Cougle, 2014) y comprobación (Coles et al., 2003; Ecker y Gönner, 2008; Pietrefesa \& Coles, 2008). Más recientemente, Cougle, Fitch, Jacobson, \& Lee (2013) llevaron a cabo tres estudios experimentales diferentes, a par- 
tir de los cuales concluyeron que la sensación de inacabado es un factor que contribuye en las conductas de comprobación compulsiva. Por su parte, en cuanto a la evitación del daño, los estudios han encontrado asociaciones menores con sintomatología compulsiva. Concretamente, en algunos estudios se halló una relación significativa entre la evitación del daño y obsesiones (Ecker, Kupfer, \& Gönner, 2014; Pietrefesa \& Coles, 2008; Taylor et al., 2014) y los síntomas de lavado/contaminación (Ecker \& Gönner, 2008), aunque de menor intensidad.

Una vez constatada la relación entre las variables objeto de estudio, se realizó un análisis Path con el objetivo de comprobar si alguna de las dos dimensiones (evitación del daño y sensación de inacabado) ejercía un rol mediador en la relación con los síntomas obsesivos. Se observó que la asociación entre la evitación del daño y los síntomas de orden y comprobación estaba mediada en gran medida por la sensación de inacabado. Este resultado apoyaría el hecho de que aunque ambas dimensiones fueron inicialmente planteadas como componentes nucleares implicados en la génesis del TOC, la sensación de inacabado podría ser más exclusiva o específica de la fenomenología TOC (como planteó Summerfeldt, 2004, 2007), mientras que la evitación del daño podría considerarse una variable con carácter transdiagnóstico, puesto que se ha observado que juega un papel importante en otros trastornos mentales diferentes al TOC (Abrams et al., 2004; Ecker, Kupfer, \& Gönner, 2014; Smith, Duffy, Stewart, Muir, \& Blackwood, 2005). Desde la perspectiva psicobiológica del temperamento de Cloninger, la evitación del daño se considera una de las cuatro dimensiones del temperamento (Cloninger, Švrakić, \& Przybeck, 1993). En un meta-análisis basado en 75 estudios se analizaron las dimensiones de temperamento propuestas por Cloninger en varios grupos de pacientes con diferentes trastornos mentales, observando que el resultado más consistente es la existencia de puntuaciones significativamente más elevadas en la dimensión de evitación del daño en todos los grupos clínicos estudiados (TOC, trastorno de pánico, fobia social, esquizofrenia, depresión mayor, trastorno bipolar y anorexia y bulimia nerviosa) en comparación con el grupo control (Miettunen y Raevuori, 2012).

Los resultados del presente estudio deben ser tomados con cautela, puesto que presenta algunas limitaciones. Dado que no se trata de un diseño experimental ni longitudinal, no se puede asegurar la relación causal entre las variables. Los resultados sugieren una direccionalidad desde el punto de vista estadístico, cuyos índices ajustan mejor. Por otra parte, a pesar de que algunos autores defienden la utilidad de llevar a cabo estudios en poblaciones análogas, en nuestro caso formada por estudiantes y familiares, para comprender los fenómenos que ocurren en el TOC (Abramowitz et al., 2014; Gibbs, 1996), los resultados derivados de esta investigación deberán confirmarse en futuros estudios contando con muestras de pacientes con TOC.

A pesar de las limitaciones mencionadas, este trabajo representa un avance en el estudio de las dimensiones de sensación de inacabado y evitación del daño y su relación con la sintomatología TOC. Los resultados aquí presenta- 
dos aportan evidencia de una mayor especificidad de la sensación de inacabado con los síntomas obsesivo-compulsivos, al menos en lo que respecta al orden y a la comprobación. No obstante, es innegable que la evitación del daño también constituye una dimensión relevante subyacente a la sintomatología obsesivocompulsiva. De hecho, esta importancia puede verse reflejada en los actuales criterios diagnósticos del TOC (DSM-5), así como la atención prestada desde los modelos cognitivo-conductuales explicativos del TOC, y en las propias manifestaciones sintomáticas de algunos pacientes. Por ello, en términos de tratamiento quizás sería más adecuado, como sugieren Cougle y Lee (2014), optar por tratamientos centrados en ambas dimensiones. Estos autores sugieren que ambas variables no tienen por qué ser excluyentes, tal vez desde la evitación del daño se puede explicar la frecuencia de los comportamientos compulsivos, mientras que desde la sensación de inacabado podría explicarse la duración y las dificultades para finalizar dichas conductas.

\section{Referencias}

Abramowitz, J. S. (1996). Variants of exposure and response prevention in the treatment of obsessive-compulsive disorder: A meta-analysis. Behavior Therapy, 27(4), 583-600. https://doi.org/10.1016/S00057894(96)80045-1

Abramowitz, J. S., Fabricant, L. E., Taylor, S., Deacon, B. J., McKay, D., \& Storch, E. A. (2014). The relevance of analogue studies for understanding obsessions and compulsions. Clinical Psychology Review, 34(3), 206-217. https://doi. org/10.1016/j.cpr.2014.01.004

Abrams, K. Y., Yune, S. K., Kim, S. J., Jeon, H. J., Han, S. J., Hwang, J., ... y Lyoo, I. K. (2004). Trait and state aspects of harm avoidance and its implication for treatment in major depressive disorder, dysthymic disorder, and depressive personality disorder. Psychiatry and Clinical Neurosciences, 58(3), 240-248. https://doi. org/10.1111/j.1440-1819.2004.01226.x

American Psychiatric Association (2013). Diagnostic and statistical manual of mental disorders (5th Edition). Washington, DC: Author.

Belloch, A., Fornés, G., Carrasco, A., LópezSolá, C., Alonso, P., \& Menchón, J. M. (2016). Incompleteness and not just right experiences in the explanation of obsessive-compulsive disorder. Psychiatry Research, 236, 1-8. https://doi.org/10.1016/j. psychres.2016.01.012

Belloch, A., Roncero, M., García-Soriano, G., Carrió, C., Cabedo, E., \& Fernández- Álvarez, H. (2013). The Spanish version of the Obsessive-Compulsive Inventory- Revised OCI-R: reliability, validity, diagnostic accuracy, and sensitivity to treatment effects in clinical samples. Journal of Obsessive-Compulsive and Related Disorder, 2(3), 249-256. https:// doi.org/10.1016/j.jocrd.2013.05.001

Bentler, P. M. (2006). EQS6.1 Structural Equations Program Manual. Multivariate Software, Inc, Encino, CA.

Bentler, P. M., Bonnet, D. G. (1980). Significance tests and goodness of fit in the analysis of covariance structures. Psychological Bulletin, 88, 588-606. https://doi.org/10.1037/00332909.88.3.588

Calamari, J. E., Cohen, R. J., Rector, N. A., SzacunShimizu, K., Riemann, B. C., \& Norberg, M. N. (2006). Dysfunctional belief-based obsessive-compulsive disorder subgroups. Behaviour Research and Therapy, 44(9), 1347-1360. https://doi.org/10.1016/j. brat.2005.10.005

Carrasco, A., y Belloch, A. (2013). Algo no está bien: una nueva lectura de la duda obsesiva. Psicología Conductual, 21(2), 341-361.

Cloninger, C. R., Svrakic, D. M., \& Przybeck, T. R. (1993). A psychobiological model of temperament and character. Archives of General Psychiatry, 50(12), 975-990. https://doi.org/10.1001/ archpsyc.1993.01820240059008 
Coles, M. E., Frost, R. O., Heimberg, R. G., \& Rhéaume, J. (2003). "Not just right experiences": perfectionism, obsessivecompulsive features and general psychopathology. Behaviour Research and Therapy, 41(6), 681-700. https://doi. org/10.1016/S0005-7967(02)00044-X

Coles, M. E., Heimberg, R. G., Frost, R. O., \& Steketee, G. (2005). Not just right experiences and obsessive-compulsive features: Experimental and self-monitoring perspectives. Behaviour Research and Therapy, 43(2), 153-167. https://doi. org/10.1016/j.brat.2004.01.002

Cougle, J. R., Fitch, K. E., Jacobson, S., \& Lee, H. J. (2013). A multi-method examination of the role of incompleteness in compulsive checking. Journal of Anxiety Disorders, 27(2), 231-239.

https://doi.org/10.1016/j. janxdis.2013.02.003

Cougle, J. R., \& Lee, H. J. (2014). Pathological and non-pathological features of obsessivecompulsive disorder: Revisiting basic assumptions of cognitive models. Journal of Obsessive-Compulsive and Related Disorders, 3(1), 12-20. https://doi. org/10.1016/j.jocrd.2013.11.002

Ecker, W., \& Gönner, S. (2008). Incompleteness and harm avoidance in OCD symptom dimensions. Behaviour Research and Therapy, 46(8), 895-904. https://doi. org/10.1016/j.brat.2008.04.002

Ecker, W., Kupfer, J., \& Gönner, S. (2014). Incompleteness as a link between obsessive-compulsive personality traits and specific symptom dimensions of obsessive-compulsive disorder. Clinical Psychology and Psychotherapy, 21(5), 394402. https://doi.org/10.1002/cpp.1842

Ferrão, Y. A., Shavitt, R. G., Prado, H., Fontenelle, L. F., Malavazzi, D. M., de Mathis, M. A., ... \& do Rosario, M. C. (2012). Sensory phenomena associated with repetitive behaviors in obsessive-compulsive disorder: an exploratory study of 1001 patients. Psychiatry Research, 197(3), 253-258. https://doi.org/10.1016/j. psychres.2011.09.017
Foa, E. B., Huppert, J. D., Leiberg, S., Langner, R., Kichic, R., Hajcak, G., \& Salkovskis, P. M. (2002). The Obsessive-Compulsive Inventory: development and validation of a short version. Psychological Assessment, 14 (4) 485-496. https://doi.org/10.1037/1040-3590.14.4.485

Foa, E. B., Liebowitz, M. R., Kozak, M. J., Davies, S., Campeas, R., Franklin, M. E., ... \& Simpson, H. B. (2005). Randomized, placebo-controlled trial of exposure and ritual prevention, clomipramine, and their combination in the treatment of obsessive-compulsive disorder. American Journal of Psychiatry, 162(1), 151-161. https:// doi.org/10.1176/appi.ajp.162.1.151

Foa, E. B., Steketee, G., \& Milby, J. B. (1980). Differential effects of exposure and response prevention in obsessivecompulsive washers. Journal of Consulting and Clinical Psychology, 48(1), 71-79. https:// doi.org/10.1037//0022-006x.48.1.71

Fornés, G., Ruiz-Fernández, M. A., y Belloch, A. (2016). Sensación de inacabado y experiencias "not just right" como motivadoras de los síntomas obsesivocompulsivos. Revista de Psicopatología y Psicología Clínica, 21, 105-118.

García-Soriano, G., Belloch, A., \& Morillo Prats, C. (2008). Sobre la heterogeneidad del trastorno obsesivo-compulsivo: una revisión. Revista de Psicopatología y Psicología Clínica, 13(2), 65-84.

Ghisi, M., Chiri, L. R., Marchetti, I., Sanavio, E., \& Sica, C. (2010). In search of specificity: "Not just right experiences" and obsessivecompulsive symptoms in non-clinical and clinical Italian individuals. Journal of Anxiety Disorders, 24(8), 879-886. https://doi. org/10.1016/j.janxdis.2010.06.011

Gibbs, N. A. (1996). Non-clinical populations in research on obsessive-compulsive disorder: A critical review. Clinical Psychology Review, 16(8), 729-773. https://doi.org/10.1016/ S0272-7358(96)00043-8

Hu, L. T., \& Bentler, P. M. (1999). Cutoff criteria for fit indexes in covariance structure analysis: Conventional criteria versus new alternatives. Structural Equation Modeling, 6(1), 1-55. https://doi. org/10.1080/10705519909540118 
Leckman, J. F., Walker, D. E., Goodman, W. K., Pauls, D. L., \& Cohen, D. J. (1994). "Just right" perceptions associated with compulsive behavior in Tourette's syndrome. American Journal of Psychiatry, 151(5), 675-680. https://doi.org/10.1176/ajp.151.5.675

Lindsay, M., Crino, R., \& Andrews, G. (1997). Controlled trial of exposure and response prevention in obsessivecompulsive disorder. The British Journal of Psychiatry, 171(2), 135-139. https://doi. org/10.1192/bjp.171.2.135

Lochner, C., \& Stein, D. J. (2003). Heterogeneity of obsessive-compulsive disorder: a literature review. Harvard Review of Psychiatry, 11(3), 113-132. https://doi. org/10.1080/10673220390217926

Miettunen, J., \& Raevuori, A. (2012). A metaanalysis of temperament in axis I psychiatric disorders. Comprehensive Psychiatry, 53(2), 152-166. https://doi.org/10.1016/j. comppsych.2011.03.008

Miguel, E. C., do Rosário-Campos, M. C., da Silva Prado, H., do Valle, R., Rauch, S. L., Coffey, B. J., ... \& Leckman, J. F. (2000). Sensory phenomena in obsessive-compulsive disorder and Tourette's disorder. The Journal of Clinical Psychiatry, 61(2), 150-156. Recuperado de https://www.psychiatrist. com/jcp/article/pages/2000/v61n02/ v61n0213.aspx

Pietrefesa, A. S., \& Coles, M. E. (2008). Moving beyond an exclusive focus on harm avoidance in obsessive compulsive disorder: Considering the role of incompleteness. Behavior Therapy, 39(3), 224-231. https://doi.org/10.1016/j. beth.2007.08.004

Pietrefesa, A. S., \& Coles, M. E. (2009). Moving beyond an exclusive focus on harm avoidance in obsessive-compulsive disorder: behavioral validation for the separability of harm avoidance and incompleteness. Behavior Therapy, 40(3), 251-259. https://doi.org/10.1016/j. beth.2008.06.003

Rachman, S. J. (2002). A cognitive theory of compulsive checking. Behaviour Research and Therapy, 40(6), 625-639. https://doi. org/10.1016/S0005-7967(01)00028-6

Radomsky, A. S., \& Rachman, S. (2004). Symmetry, ordering and arranging compulsive behaviour. Behaviour Research and Therapy, 42(8), 893-913. https://doi. org/10.1016/j.brat.2003.07.001

Rapoport, J. L. (1991). Basal ganglia dysfunction as a proposed cause of obsessive-compulsive disorder. Psychopathology and The Brain, 7795. https://doi.org/10.1038/tp.2011.5

Rasmussen, S.A., \& Eisen, J.L. (1992). The epidemiology and clinical features of obsessive compulsive disorder. The Psychiatric Clinics of North America, 15, 743-758. https://doi.org/10.1016/S0193953X(18)30205-3

Salkovskis, P. M. (1999). Understanding and treating obsessive-compulsive disorder. Behaviour Research and Therapy, 37(Suppl. 1), S29-S52. https://doi.org/10.1016/S00057967(99)00049-2

Smith, D. J., Duffy, L., Stewart, M. E., Muir, W. J., \& Blackwood, D. H. (2005). High harm avoidance and low self-directedness in euthymic young adults with recurrent, early-onset depression. Journal of Affective Disorders, 87(1), 83-89. https://doi. org/10.1016/j.jad.2005.03.014

Summerfeldt, L. J. (2004). Understanding and treating incompleteness in obsessive compulsive disorder. Journal of Clinical Psychology, 60(11), 1155-1168. https://doi. org/10.1002/jclp.20080

Summerfeldt, L. J. (2007). Treating incompleteness, ordering, and arranging concerns. In: M. M. Antony, C. Purdon, \& L. J. Summerfeldt (Eds.), Psychological treatment of obsessive-compulsive disorder (pp. 187-207). Washington, DC: American Psychological Association.

Summerfeldt, L. J., Kloosterman, P. H., Antony, M. M., \& Swinson, R. P. (2014). Examining an obsessive-compulsive core dimensions model: Structural validity of harm avoidance and incompleteness. Journal of ObsessiveCompulsive and Related Disorders, 3(2), 83-94. https://doi.org/10.1016/j.jocrd.2014.01.003 
Summers, B. J., Fitch, K. E., \& Cougle, J. R. (2014). Visual, tactile, and auditory "not just right" experiences: associations with obsessive-compulsive symptoms and perfectionism. Behavior Therapy, 45(5), 678-689. https://doi.org/10.1016/j. beth.2014.03.008

Tallis, F. (1996). Compulsive washing in the absence of phobic and illness anxiety. Behaviour Research and Therapy, 34(4), 361-362. https://doi org/10.1016/00057967(95)00079-8

Taylor, S., McKay, D., Crowe, K. B., Abramowitz, J. S., Conelea, C. A., Calamari, J. E., \& Sica, C. (2014). The sense of incompleteness as a motivator of obsessive-compulsive symptoms: An empirical analysis of concepts and correlates. Behavior Therapy, 45(2), 254-262. https://doi.org/10.1016/j. beth.2013.11.004

Tolin, D. F., Abramowitz, J. S., Kozak, M. J., \& Foa, E. B. (2001). Fixity of belief, perceptual aberration, and magical ideation in obsessive-compulsive disorder. Journal of Anxiety Disorders, 15(6), 501-510. https:// doi.org/10.1016/S0887-6185(01)00078-0

Whittal, M. L., Robichaud, M., Thordarson, D. S., \& McLean, P. D. (2008). Group and individual treatment of obsessive-compulsive disorder using cognitive therapy and exposure plus response prevention: A 2-year follow-up of two randomized trials. Journal of Consulting and Clinical Psychology, 76(6), 1003-1014. https://doi.org/10.1037/a0013076 